\title{
Variability in Risk Assessment and Management Plan (RAMP) scores completed as part of the Ontario Johne's Education and Management Assistance Program (2010-2013)
}

\author{
Laura Pieper, ${ }^{*}$ Trevor J. DeVries,† Ulrike S. Sorge,‡ Ann Godkin,§ Karen J. Hand,\# Nicole R. Perkins, ${ }^{*}$ \\ Jamie Imada, ${ }^{*}$ and David F. Kelton*1 \\ *Department of Population Medicine, and \\ †Department of Animal and Poultry Science, University of Guelph, Ontario, Canada, N1G 2W1 \\ $\ddagger$ Department of Veterinary Population Medicine, University of Minnesota, St. Paul 55108 \\ §Veterinary Science and Policy Group, Ontario Ministry of Agriculture and Food/Ministry of Rural Affairs (OMAF/MRA), Ontario, Canada, N1G 4Y2 \\ \#Strategic Solutions Group, Puslinch, Ontario, Canada, NOB 2J0
}

\section{ABSTRACT}

As a proactive measure toward controlling the nontreatable and contagious Johne's disease in cattle, the Ontario dairy industry launched the voluntary Ontario Johne's Education and Management Assistance Program in 2010. The objective of this study was to describe the results of the first $4 \mathrm{yr}$ of the program and to investigate the variability in Risk Assessment and Management Plan (RAMP) scores associated with the county, veterinary clinic, and veterinarian. Of 4,158 Ontario dairy farms, $2,153(51.8 \%)$ participated in the program between January 2010 and August 2013. For this study, RAMP scores and whole-herd milk or serum ELISA results were available from 2,103 farms. Herdlevel ELISA-positive prevalence (herds with one or more test-positive cows were considered positive) was $27.2 \%$. Linear mixed model analysis revealed that the greatest RAMP score variability was at the veterinarian level $(24.2 \%)$, with relatively little variability at the county and veterinary clinic levels. Consequently, the annual RAMP should be done by the same veterinarian to avoid misleading or discouraging results.

Key words: paratuberculosis, risk assessment, control program, participation

\section{INTRODUCTION}

Johne's disease (JD) or paratuberculosis is a chronic infectious disease of ruminants associated with granulomatous enteritis caused by Mycobacterium avium ssp. paratuberculosis (MAP). The most common route of disease transmission is by ingestion of contaminated feces or milk in the first days after birth. The risk of

Received September 3, 2014.

Accepted December 19, 2014

${ }^{1}$ Corresponding author: dkelton@uoguelph.ca developing JD is especially high for calves exposed at calving in a highly contaminated calving area (Windsor and Whittington, 2010).

Estimates of the apparent JD herd-level seroprevalence in dairy herds among Canadian provinces vary between 33 and $74 \%$ (Tiwari et al., 2006). Diagnostic and screening tests usually have low sensitivity compared with tissue culture or fecal culture as the gold standard, especially when the prevalence in the herd is low or the individual is shedding few bacteria (Tiwari et al., 2006). Therefore, test-and-cull programs alone are considered less effective than risk-based prevention strategies for JD control (Kudahl et al., 2008). Risk prevention strategies aim to reduce the risk of disease transmission through improvements in targeted management practices, including calving and calf rearing management, hygiene, and biosecurity.

For farm-level delivery of JD control programs, trained veterinarians commonly evaluate animal management practices using a standardized risk assessment instrument. Based on that, they decide with the producer how to implement best management practices for JD control. Although attempts have been made to standardize the risk assessments used, they may include subjective measurements and observations (e.g., hygiene scoring) that could be influenced by the perspective and biases of the assessing veterinarian. Additionally, specific veterinary clinic policies or regional differences in the diversity of dairy herds might introduce variability at those levels that are not attributable to the farm situation itself. Berghaus et al. (2005) reported a state-level variability in the risk assessment of $5 \%$ and variability among assessors of $15 \%$.

To decrease the effect of JD in Canadian dairy herds, the Canadian Johne's Disease Initiative was created as a collaborative effort of dairy industry, producer organizations, governments, and veterinary schools. Within the initiative, most provinces have established an in- 
dividual JD control program (Barker et al., 2012). In the province of Ontario, the voluntary Ontario Johne's Education and Management Assistance Program (OJEMAP) was launched in January 2010 (www. johnes.ca). The risk assessment used in the OJEMAP was adapted from that used by Sorge et al. (2011) and is based on identified high-risk areas for MAP transmission. The aforementioned authors showed that farms participating in the risk assessment-based control program could reduce their risk scores as well as the apparent within-herd milk ELISA prevalence for JD. Full implementation of the OJEMAP was completed in August 2013. A detailed description of program results is necessary to provide information for researchers and government officials for improvement and development of JD control programs.

Therefore, the objectives of this study were (1) to describe the participation, risk assessment scores, recommendations, and ELISA prevalence in the OJEMAP, and (2) to assess the variability of risk assessment scores associated with the county, veterinary clinic, veterinarian, and herd.

\section{MATERIALS AND METHODS}

\section{Ontario Johne's Education and Management Assistance Program}

A standardized management questionnaire, the Risk Assessment and Management Plan (RAMP), was administered by trained, private veterinarians. The RAMP was a semiquantitative tool, with low scores indicating a low risk of disease transmission. It contained 5 risk assessment sections: (1) general JD management and biosecurity (maximum 60 points); (2) calving area risk management (maximum 80 points); (3) preweaned heifer management (maximum 70 points); (4) postweaned heifer management (maximum 40 points); and (5) adult cow and manure management (maximum 50 points). The maximum overall RAMP score combining all 5 sections was 300 points. Farm-specific recommendations for management changes to reduce MAP transmission in high-risk areas were made by the veterinarian. Expenses for the assessing veterinarian to administer the RAMP were covered by the farmers. Through the OJEMAP, more than $95 \%$ of Ontario dairy veterinarians have been trained and registered to conduct the risk assessment for their clients. A RAMP manual was provided to veterinarians for further consistency of assessments. The RAMP and the RAMP manual can be found on the program webpage (www. johnes.ca).

Testing of milk or serum from all lactating cows on a single date was used to identify MAP antibody
ELISA-positive cows. For sample management, all counties were assigned a 40-d window for enrollment in the program. For herds participating in milk recording through CanWest DHI, milk was collected from each cow through milk recording meters and submitted to the DHI laboratory for automated analysis. The Parachek milk ELISA (Prionics AG, Schlieren, Switzerland) used in the program was found to have a sensitivity of $61.1 \%$ and a specificity of $94.7 \%$ compared with fecal culture (Hendrick et al., 2005a). Cows were considered negative at a corrected optical density $($ OD) $<0.00$, suspicious at OD 0.00 to 0.09 , positive at OD 0.1 to 0.99 , or high positive $(\mathbf{H P})$ at $\mathrm{OD} \geq 1.0$. For herds not participating in milk recording or for additional dry cow testing, serum samples taken by the herd veterinarian were submitted to the Animal Health Laboratory (University of Guelph, ON, Canada) for analyses using the Idexx HerdChek Mycobacterium paratuberculosis ELISA (Idexx Laboratories, Westbrook, ME). Cows were considered negative at a sample-to-positive control ratio (S:P) $<0.10$, suspicious at S:P 0.10 to 0.24 , positive at $\mathrm{S}: \mathrm{P} 0.25$ to 0.99 , or $\mathrm{HP}$ at $\mathrm{S}: \mathrm{P} \geq 1.0$, and the sensitivity and specificity compared with fecal culture were 73.6 and $87.5 \%$, respectively (Hendrick et al., 2005a). When cows with HP ELISA test results were identified, farmers were encouraged to dispose of these animals within $90 \mathrm{~d}$ of testing, ensuring that they were not moved to another dairy herd or into the human food chain. Expenses for the ELISA testing (Can\$8/ cow) and compensation for cow removal (Can $\$ 500 /$ disposed HP cow) were covered by the program for every farm participating for the first time, if HP cows were removed in time. In 2013, all farms were offered a second program-funded test.

\section{Data}

The data set was extracted from the OJEMAP database and included RAMP completed between January 2010 and August 2013 and the corresponding herd Johne's ELISA test results. All but the first duplicate observations (repeated observations, because some herds had tested more than once) and herds that included only a RAMP score or an ELISA test result, but not both, were removed. A flowchart of data management is shown in Figure 1. Information about the county in which the herd was located, the name of the veterinarian who completed the RAMP and their veterinary clinic, number of cows tested with either milk or serum ELISA, number of ELISA-positive cows, number of ELISA HP cows, the HP disposal method, the overall RAMP score and section scores, and the recommendations given to the producers were available for each farm. For this study, a farm was considered JD 


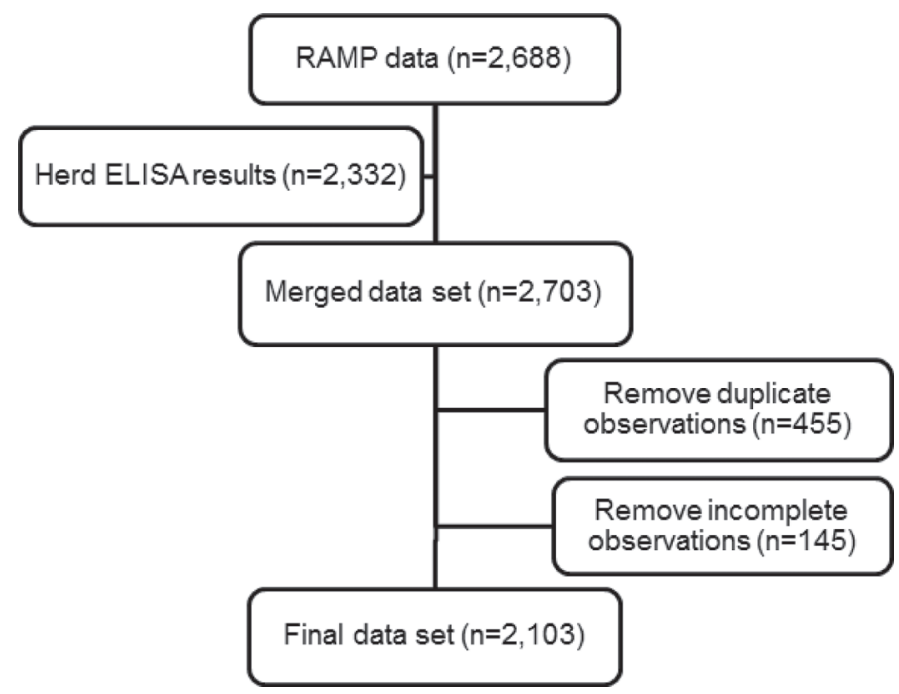

Figure 1. Flowchart of number of observations at each stage from combining the Risk Assessment and Management Plan (RAMP) data with the ELISA data to the final number of observations analyzed.

herd ELISA status (HES)-positive if they had at least one cow with a JD ELISA positive or HP result; JD ELISA suspect cows were considered negative for this analysis.

The recommendations given to the farmers after the RAMP were coded with numerical codes by a single person (J. I.). For this study, the recommendations were manually associated with the respective RAMP questions and sections in the RAMP based on reference of recommendations to risk described and assessed in this area.

\section{Statistical Analyses}

The RAMP section scores were calculated by adding the respective question scores in each section. Normality of the data was assessed visually using histograms. For descriptive statistics, the geometric mean was reported for non-normally distributed data (e.g., section scores). A small number (1) was added to variables that included null values to allow for proper transformation.

The relationship between overall RAMP score and number of test-positive cows was nonlinear. Logarithmic transformation did not improve linearity and, consequently, the models were built with the dichotomous variable HES ( 1 = one or more ELISA-positive animals; $0=$ zero ELISA-positive animals). This variable was included in the model to account for differences in JD herd ELISA prevalence among veterinarians, veterinary clinics, or regions.

A hierarchical approach to model building was started with a linear regression model with RAMP score as the outcome and HES as the fixed effect. Additionally, year of assessment was tested as a categorical fixed effect. Each level of the hierarchy (i.e., veterinarian, veterinary clinic, county) was first tested alone as a random effect for explanation of RAMP score variability. Then, starting at the veterinarian level, higher levels (veterinary clinic and county) were offered to the previous model as random effects. A random slope was tested to determine whether the strength of the relationship between HES and RAMP score was dependent on the assessing veterinarian. Bayesian information criterion and likelihood ratio tests were used to find the best model. The model fit was assessed graphically at each hierarchical level by plotting the residuals against the predicted value and the inverse normal score.

The statistical data analyses were conducted using STATA 10.1 (StataCorp, College Station, TX). A probability level of $P<0.05$ was considered significant. Geographical maps displaying the number of herds per county and the proportion of herds participating per county were generated using ArcMap 10 (ESRI, Redlands, CA).

\section{RESULTS}

Fifty-two percent of all herds in all counties in Ontario participated in the program, but the proportion of participating herds per county varied between 8.8 and $86.1 \%$. Areas with high participation were not necessarily areas with high herd density (Figure 2). After data cleaning, 2,103 herd observations with complete RAMP information and ELISA results were retained in the data set. There was considerable variation in program participation at the county, veterinary clinic, and veterinarian levels (Table 1 ). Some veterinarians conducted up to 63 RAMP, whereas others conducted only 1 . Of all tested farms, $572(27.2 \%)$ had at least one JD ELISA-positive cow. Of these, $261(12.4 \%)$ had at least 2 JD ELISA-positive cows, and $130(6.2 \%)$ had at least one HP cow in their herd. Of 106 farms on which the HP disposal method was noted, 19 (18\%) buried, $24(23 \%)$ composted, and $63(59 \%)$ used a deadstock service to dispose of their HP animals. The average number of JD ELISA-positive cows and the average within-herd ELISA prevalence was 0.34 (95\% CI: 0.31 to 0.37 ) and $0.85 \%$ (95\% CI: 0.76 to $0.94 \%$ ) for all tested herds, and 1.93 (95\% CI: 1.80 to 2.07 ) and $3.16 \%$ (95\% CI: 2.93 to $3.40 \%$ ) for HES-positive herds, respectively. The average overall RAMP score was 121.3 (95\% CI: 119.7 to 122.8 ) on all and 129.8 (95\% CI: 126.8 to 132.8 ) on HES-positive farms. Section 1 score was clearly bimodal, with many farmers having a zero value, indicating that the risk of introducing JD to the farm was very low. Therefore, neither an arithmetic mean nor a geometric mean as a measure of central 


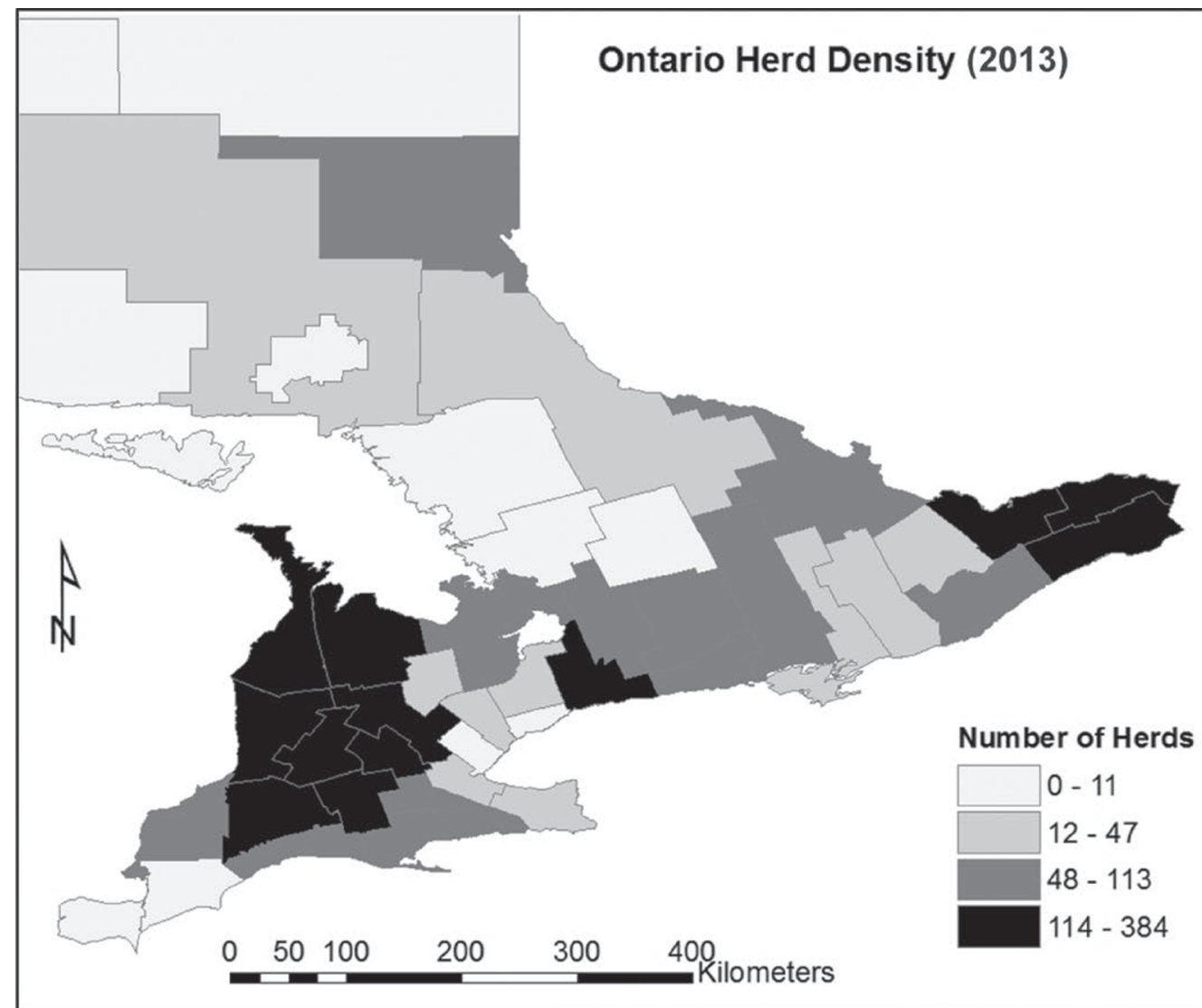

\section{Percent of Herds in County Participating in Ontario Johne's Program}

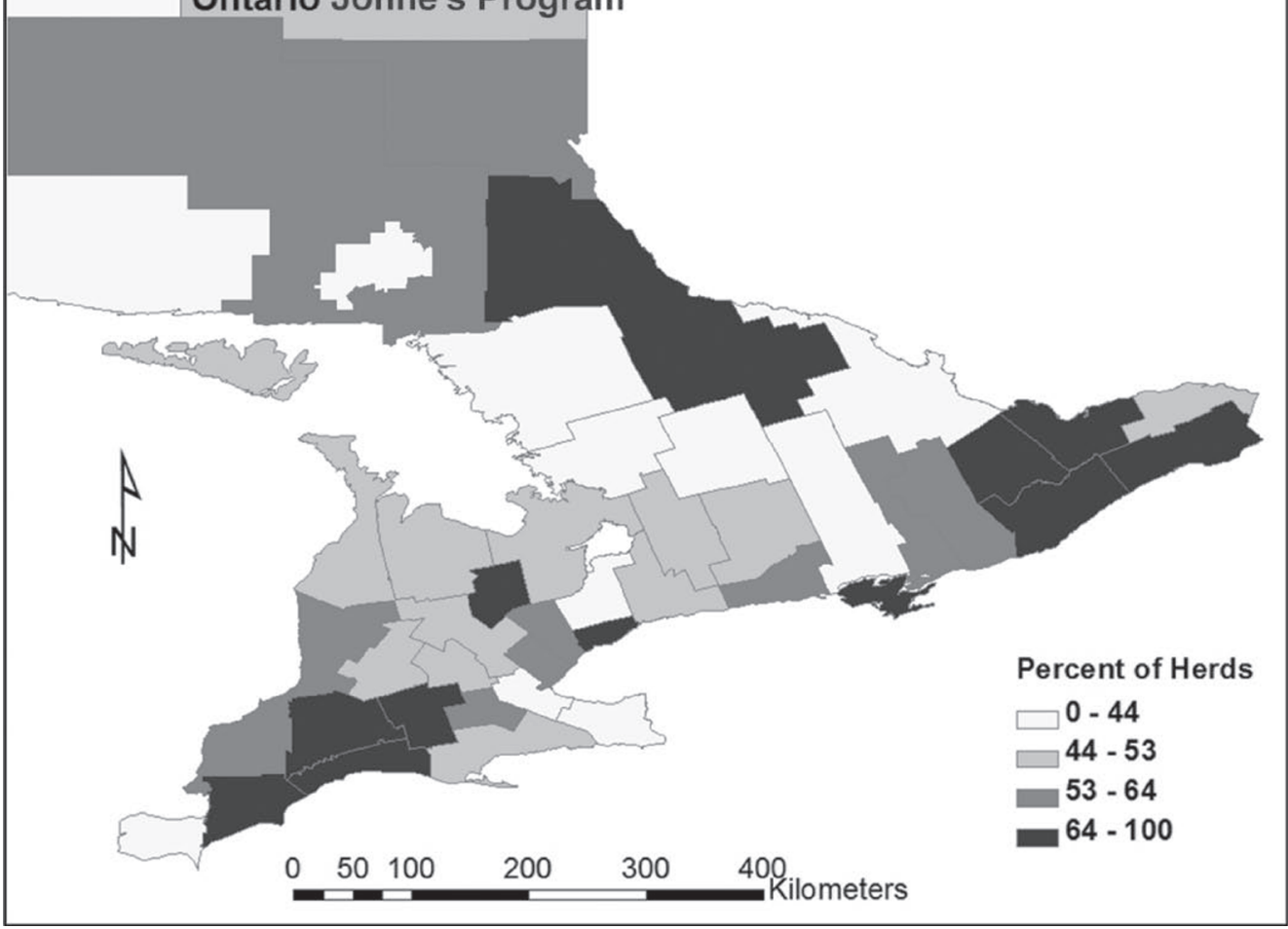

Figure 2. Choropleth maps of total number of dairy herds per county in 2013 and proportion of herds per county participating in the Ontario Johne's Education and Management Assistance Program in southern Ontario from January 2010 to August 2013. 
Table 1. Distribution of Risk Assessment and Management Plans (RAMP) at the county, veterinary clinic, and veterinarian level

\begin{tabular}{lccccc}
\hline Level & $\begin{array}{c}\text { No. of } \\
\text { categories }\end{array}$ & $\begin{array}{c}\text { Mean no. } \\
\text { of RAMP }\end{array}$ & $95 \%$ CI & Minimum & Maximum \\
\hline County & 47 & 24.7 & $17.2-35.5$ & 1 & 187 \\
Veterinary clinic & 107 & 9.1 & $7.0-11.7$ & 1 & 122 \\
Veterinarian & 184 & 7.7 & $6.7-8.9$ & 1 & 63 \\
\hline${ }^{1}$ Ceometrim
\end{tabular}

${ }^{1}$ Geometric mean.

tendency describes the data appropriately. The proportion of herds with a section 1 score of zero was $27.5 \%$ in all herds and $20.8 \%$ in HES-positive herds. Detailed descriptions of RAMP scores, number of cows tested, and within-herd ELISA prevalence for all herds and JD HES-positive herds are given in Table 2.

After completing the initial risk assessment, veterinarians gave $0(0.5 \%), 1(10.0 \%), 2(24.6 \%)$, or 3 (64.9\%) recommendations to the farmers (mean: 2.54, median: 3) for changes to improve JD control on their farm. The most common recommendation, with $44.1 \%$ of participating farmers receiving it, was to not purchase cows, to minimize purchases, or to buy only from known low-risk herds. Table 3 displays the 14 most common recommendations that were each given to over $5 \%$ of herds.

When hierarchy (i.e., county, veterinary clinic, veterinarian) was tested as the only random effect, 5,19 , and $24 \%$ of the RAMP total score was explained by county, veterinary clinic, and veterinarian, respectively. Any additional variation in the RAMP score explained at the county or veterinary clinic level was not significant when veterinarian was in the model. The random slope did not contribute to the explanation of variation in the model. Confounding by veterinarian was not detected, and year of assessment was not significant in the model. The RAMP score was 11.7 points higher on HES-positive farms than on negative farms (Table 4). In the final model, the likelihood ratio test (against a linear model without random effects) was highly significant $(P<$ 0.001 ), and residuals at both levels were normally distributed and homoscedastic, indicating good model fit.

\section{DISCUSSION}

This paper describes results from the voluntary Johne's disease control program in Ontario, Canada. Of 2,103 herds included in this analysis, $27.2 \%$ had at least one JD milk ELISA-positive animal. This estimate is lower than the $34 \%$ (95\% CI: 21 to $47 \%$ ) milk ELISA prevalence estimate reported by Hendrick et al. (2005b) using the same cut-off. One might speculate that increased educational efforts through the program could have reduced the herd ELISA prevalence since the aforementioned study. However, the confidence intervals in the 2 studies are quite wide and do overlap, indicating that the estimates are not significantly different. The herd-level JD ELISA-positive prevalence might be overestimated due to the specificity of the test being less than $100 \%$.

The most common recommendation made by veterinarians to dairy farmers (44\%) was to keep their herd closed or to be more cautious when purchasing animals. This may indicate a perception of veterinarians that producers potentially have insufficient screening and isolation practices when purchasing animals and they should therefore avoid bringing cattle in, especially if they are low prevalence herds. Introduction of cattle into the herd has been identified as a risk factor for JD herd positive results in other studies (Wells and Wagner, 2000; Sorge et al., 2012). In the current study, $72.5 \%$ of producers had a nonzero score in section 1, showing that they had some form of herd additions within the last 5 yr. On the other hand, 21\% of HES-positive producers had a zero score in section 1, indicating that they had not purchased animals. However, this measure does not account for earlier herd additions, and herds may have introduced infectious cattle more than $5 \mathrm{yr}$ ago. Consequently, Ontario dairy farmers might have benefitted from a larger educational campaign to increase awareness regarding risks of disease introduction when purchasing animals (buyer beware; Godkin, 2011; Kelton, 2014).

The RAMP score was 12 points higher on HESpositive farms compared with HES-negative farms. One question in the risk assessment specifically refers to the HES (question 5 in section 2: Calving area used by JD clinical or test-positive cows?) with the answer categories $1,4,7$, or 10 , based on increasing JD prevalence. However, this question alone could not account for the observed RAMP score difference between HES-positive and HES-negative herds. This suggests that there is a positive association between positive HES and RAMP score beyond this specific question.

The county-level intraclass correlation coefficient in our study was very low (5\%), and is similar to the estimate by Berghaus et al. (2005) of the state-level correlation in the United States. Furthermore, variation at 
Table 2. Description of ELISA test results and Risk Assessment and Management Plan (RAMP) scores in all herds and herd ELISA status (HES)-positive herds participating in the Ontario Johne's Education and Management Assistance Program

\begin{tabular}{|c|c|c|c|c|c|c|c|c|}
\hline \multirow[b]{2}{*}{ Item } & \multicolumn{4}{|c|}{ All herds $(\mathrm{n}=2,103)$} & \multicolumn{4}{|c|}{ HES-positive herds $(\mathrm{n}=572)$} \\
\hline & Mean $^{1}$ & $95 \% \mathrm{CI}$ & Minimum & Maximum & Mean $^{1}$ & $95 \% \mathrm{CI}$ & Minimum & Maximum \\
\hline No. of cows tested & 54.1 & $52.9-55.4$ & 12 & 986 & 74.2 & $70.3-78.4$ & 18 & 986 \\
\hline RAMP score ${ }^{2}$ & 121.3 & $119.7-122.8$ & 26 & 231 & 129.8 & $126.8-132.8$ & 28 & 220 \\
\hline Section 1 score $^{3}$ & 14.9 & $13.7-16.1$ & 0 & 60 & 20.2 & $17.6-23.1$ & 0 & 60 \\
\hline Section 2 score $^{4}$ & 30.8 & $30.3-31.4$ & 8 & 74 & 33.2 & $32.2-34.3$ & 8 & 74 \\
\hline Section 3 score $^{5}$ & 19.4 & $19.0-19.9$ & 7 & 58 & 19.9 & $19.1-20.8$ & 7 & 58 \\
\hline Section 4 score $^{6}$ & 14.6 & $14.2-14.9$ & 4 & 40 & 13.9 & $13.3-14.6$ & 4 & 40 \\
\hline Section 5 score $^{7}$ & 13.7 & $13.4-14.1$ & 4 & 45 & 15.5 & $14.7-16.3$ & 4 & 44 \\
\hline No. of ELISA-positive cows & 0.34 & $0.31-0.37$ & 0 & 33 & 1.93 & $1.80-2.07$ & 1 & 33 \\
\hline Within-herd prevalence $^{8}$ of ELISA-positive cows (\%) & 0.85 & $0.76-0.94$ & 0 & 22.6 & 3.16 & $2.93-3.40$ & 0.3 & 22.6 \\
\hline No. of ELISA high-positive cows & 0.052 & $0.043-0.062$ & 0 & 5 & 0.21 & $0.17-0.24$ & 0 & 5 \\
\hline Within-herd prevalence ${ }^{8}$ of ELISA high-positive cows (\%) & 0.11 & $0.08-0.13$ & 0 & 8.7 & 0.40 & $0.32-0.48$ & 0 & 8.7 \\
\hline
\end{tabular}

\section{${ }^{1}$ Geometric mean if not indicated otherwise.}

${ }^{2}$ Maximum possible score is 300; arithmetic mean is displayed.

${ }^{3}$ Section $1=$ general Johne's Disease risk management (maximum $=60$ points).

${ }^{4}$ Section $2=$ calving area risk management (maximum $=80$ points).

${ }^{5}$ Section $3=$ heifers - preweaned risk management (maximum $=70$ points).

${ }^{6}$ Section $4=$ heifers - weaned to first calving risk management (maximum $=40$ points).

${ }^{7}$ Section $5=$ cows - risk management (maximum $=50$ points).

${ }^{8}$ Calculated as the number of (high) positive animals/number of cows tested in a herd. 
Table 3. Most frequent recommendations for Johne's disease prevention and control given by veterinary assessors to producers after the Risk Assessment and Management Plan (RAMP)

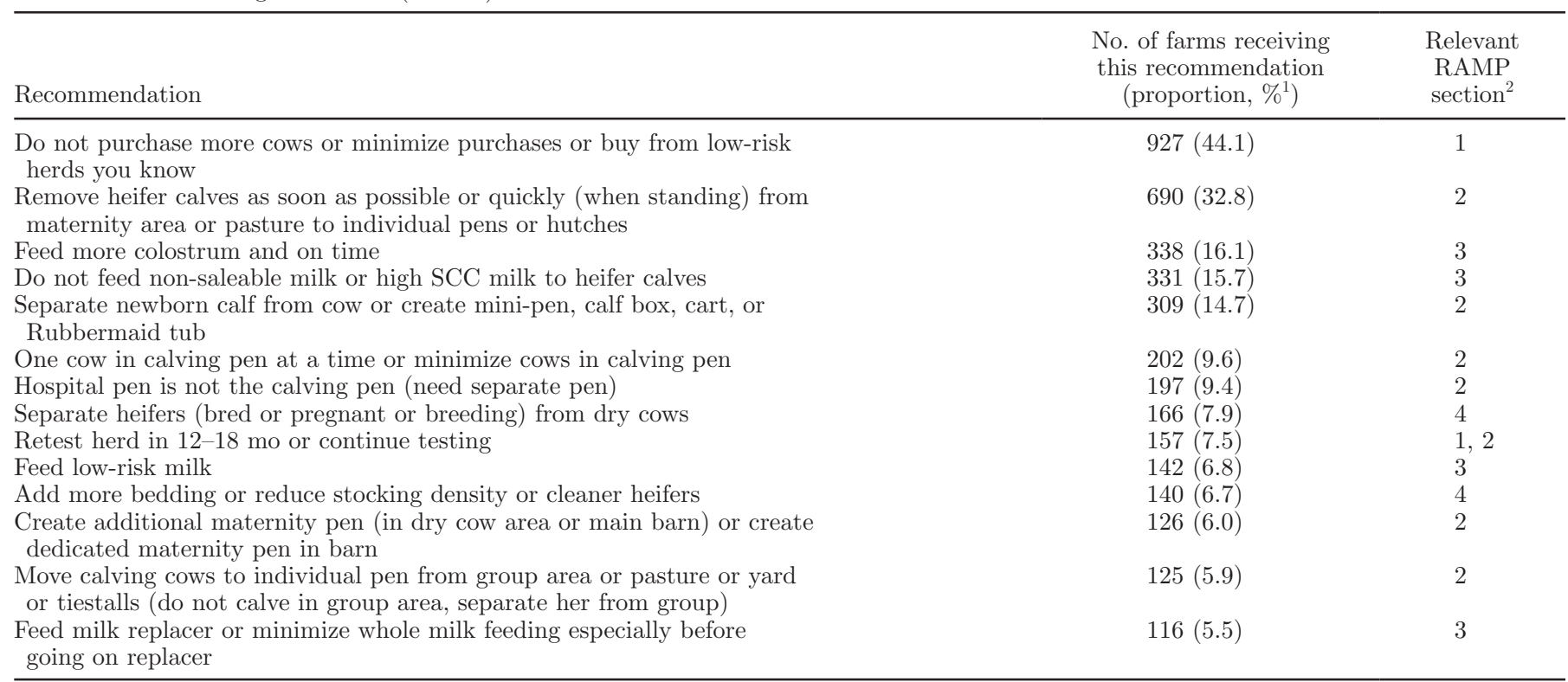

${ }^{1}$ Percentages do not add up to 100 because farmers could be given up to 3 recommendations.

${ }^{2}$ Section 1 = general Johne's disease risk management; section $2=$ calving area risk management; section $3=$ heifers - preweaned risk management; section 4 = heifers - weaned to first calving risk management; section 5 = cows - risk management.

the veterinary clinic level was explained by the respective individual veterinarians. This indicates that there was no similarity in RAMP scores among veterinarians from the same clinic. Variation at the veterinarian level was about $24 \%$, indicating that RAMPs conducted by one veterinarian are more similar to each other than to those from other veterinarians. This estimate is higher than the estimate from Berghaus et al. (2005), who reported $15 \%$ variation from the National Animal Health Monitoring System (NAHMS) Dairy 2002 study. However, NAHMS data collection was completed within a 7-mo period and, due to the random selection of farms, it is likely that the assessors (federal or state veterinary officers or animal health technicians) did not have an established relationship with the farms. One might hypothesize for our population that herds served by one veterinarian are more similar to each other compared with herds visited by other veterinarians, due to the veterinary-client relationship that might foster unique and consistent advice to each veterinarian's client herds. It could also be that one veterinarian gave generally higher or lower scores for a specific area than other veterinarians based on his or her perceptions, beliefs, and biases. However, only the random intercept, not random slope, was significant. That indicates that the association between RAMP score and HES was consistent, regardless of the assessing veterinarian. For the program, it might be necessary to provide continuing education on conducting the on-farm RAMP to minimize veterinary-level variability. Nevertheless, for herd comparisons or repeated assessments, the detected variability in the RAMP scores might cause misleading results in cases in which the farmer did not implement changes but, because the assessor changed, a positive change is documented in the RAMP. Conversely, results might be disappointing or discouraging if the producer implemented changes but, because the assessing veterinarian changed, the efforts were not recognized by a lower RAMP score. Consequently, when assessments of management improvement are done repeatedly and are used to document improvement or change, all assessments for a given farm should be done by the same veterinarian to avoid misleading results.

We found a high degree of variability in the intensity of program participation among counties, veterinary

Table 4. Final linear mixed regression model predicting the overall RAMP score in the herd ${ }^{1}$

\begin{tabular}{lccc}
\hline Item & Coefficient & $\mathrm{SE}$ & $P$-value \\
\hline Intercept & 117.86 & 1.626 & $<0.001$ \\
HES-positive farm ${ }^{2}$ & 11.72 & 1.599 & $<0.001$ \\
& Variance & $\mathrm{SE}$ & \\
\cline { 2 - 3 } & 308.90 & 45.24 & \\
Veterinarian $(\mathrm{n}=184)$ & 967.13 & 31.15 & \\
Herd $(\mathrm{n}=2,103)$ &
\end{tabular}

${ }^{1}$ Herds cluster by assessing veterinarian (intraclass correlation coefficient $=0.24$ ).

${ }^{2} \mathrm{HES}=$ herd ELISA status 
clinics, and veterinarians. Some veterinarians assessed considerably more farms than others. It might be that very active veterinarians perceive Johne's disease as a greater problem among their clients than others. It could also be that producers in those areas with low participation were less aware of the program and did not ask their veterinarians for this service. Moreover, there was anecdotal evidence of DHI technicians actively enrolling clients into the program in some regions, which might have additionally increased participation and caused differences among counties. Nevertheless, education for Johne's disease prevention and advertisement for participation in a control program should be intensified at the different levels.

\section{CONCLUSIONS}

The RAMP scores were higher for JD ELISA-positive herds than for negative herds. Considerable variation in RAMP scores was found at the level of the veterinarian but not at the clinic or county level. Risk Assessment and Management Plans from the same veterinarian were more similar to each other than to those from other veterinarians, and consecutive assessments in one farm should be done by the same veterinarian. Regional participation rates and the different involvement of veterinarians in the program indicate that educational advertisement on different levels is necessary to improve overall program participation.

\section{ACKNOWLEDGMENTS}

This study was kindly funded by the Ontario Ministry of Agriculture and Food (OMAF; Guelph, ON, Canada)-University of Guelph (Guelph, ON, Canada) research partnership.

\section{REFERENCES}

Barker, R. A., H. W. Barkema, G. Fecteau, G. P. Keefe, and D. F. Kelton. 2012. Johne's disease control in Canada - Coordinated nationally, delivered provincially. Pages 41-42 in Proc. 3rd ParaTB Forum, Sydney, Australia. IDF Bull. No. 460. International Dairy Federation, Brussels, Belgium.

Berghaus, R. D., J. E. Lombard, I. A. Gardner, and T. B. Farver. 2005. Factor analysis of a Johne's disease risk assessment questionnaire with evaluation of factor scores and a subset of original questions as predictors of observed clinical paratuberculosis. Prev. Vet. Med. 72:291-309.

Godkin, A. 2011. Buyer beware: Johne's-positive cows showing up. Milk Producer April 2011:12.

Hendrick, S., T. Duffield, D. Kelton, K. Leslie, K. Lissemore, and M. Archambault. 2005a. Evaluation of enzyme-linked immunosorbent assays performed on milk and serum samples for detection of paratuberculosis in lactating dairy cows. J. Am. Vet. Med. Assoc. 226:424-428.

Hendrick, S., T. Duffield, K. Leslie, K. Lissemore, M. Archambault, and D. Kelton. 2005b. The prevalence of milk and serum antibodies to Mycobacterium avium subspecies paratuberculosis in dairy herds in Ontario. Can. Vet. J. 46:1126-1129.

Kelton, D. F. 2014. Buyer beware-Emerging diseases coming to Canadian dairy farms. Western Canadian Dairy Seminar. Adv. Dairy Technol. 26:361-364.

Kudahl, A. B., S. S. Nielsen, and S. Østergaard. 2008. Economy, efficacy, and feasibility of a risk-based control program against paratuberculosis. J. Dairy Sci. 91:4599-4609.

Sorge, U. S., K. Lissemore, A. Godkin, J. Jansen, S. Hendrick, S. Wells, and D. F. Kelton. 2011. Changes in management practices and apparent prevalence on Canadian dairy farms participating in a voluntary risk assessment-based Johne's disease control program. J. Dairy Sci. 94:5227-5237.

Sorge, U. S., K. Lissemore, A. Godkin, J. Jansen, S. Hendrick, S. Wells, and D. F. Kelton. 2012. Risk factors for herds to test positive for Mycobacterium avium ssp. paratuberculosis-antibodies with a commercial milk enzyme-linked immunosorbent assay (ELISA) in Ontario and Western Canada. Can. Vet. J. 53:963-970.

Tiwari, A., J. A. VanLeeuwen, S. L. B. McKenna, G. P. Keefe, and H. W. Barkema. 2006. Johne's disease in Canada Part I: Clinical symptoms, pathophysiology, diagnosis, and prevalence in dairy herds. Can. Vet. J. 47:874-882.

Wells, S. J., and B. A. Wagner. 2000. Herd-level risk factors for infection with Mycobacterium paratuberculosis in US dairies and association between familiarity of the herd manager with the disease or prior diagnosis of the disease in that herd and use of preventive measures. J. Am. Vet. Med. Assoc. 216:1450-1457.

Windsor, P. A., and R. J. Whittington. 2010. Evidence for age susceptibility of cattle to Johne's disease. Vet. J. 184:37-44. 